\title{
MODELING BIOTERRORISM PREPAREDNESS WITH SIMULATION IN RURAL HEALTHCARE SYSTEM
}

\author{
Lisa Patvivatsiri
}

\author{
Production Modeling Corporation (PMC) \\ 15726 Michigan Ave \\ Dearborn, M.I. 48126, U.S.A.
}

\author{
Elliot J. Montes, Jr.
}

\author{
W. L. Gore and Associate \\ 1505 N. Fourth St. \\ Flagstaff, A.Z. 86004, U.S.A
}

\author{
Ouyang Xi \\ American Bureau of Shipping \\ 16855 Northchase Dr. \\ Houston, T.X. 77060, U.S.A
}

\begin{abstract}
As a result of the anthrax letter incidents in 2001, concerns about terrorists' use of biological agents have increased dramatically at government state and federal levels. The problem of providing sufficient resources in a healthcare system during a widespread bioterrorist attack is important issues all hospitals encounter. The innovative computer simulation models of the overall treatment process at Lubbock area healthcare systems were developed using the professional simulation software Flexsim 2.6 to determine the total time patient stays in the system and to identify the staff requirement in order to avoid delays in treatment for a variety of hypothetical bioterrorist attack scenarios.
\end{abstract}

\section{INTRODUCTION}

As the vulnerabilities exist towards terrorist attack, the U.S. government and local leaders are assessing their preparedness to cope with terrorism. Healthcare systems, as one of the key public infrastructures, have raised more concerns to the society. More recently, many papers and articles pertaining to bioterrorism responses have been published to give guidelines for healthcare system preparedness. In addition, modern operations research, system management, and technology techniques have been applied to the outbreak and bioterrorism response problem. Among various techniques, simulation modeling allows users to reconstruct a more comprehensive representation of realworld features during disaster response. For example, this modeling can represent attribute-dependent and timevarying paths for different entities competing for a limited set of resources (Welter et al., 2001). Since simulation is a powerful tool for analyzing and improving operations without disturbing existing systems, this tool has been used to support operational decision-making in response to a variety of bioterrorist threats. Simulation models have been employed to estimate the impact of large-scale biological attacks and to design or select appropriate response strategies.
Data from these studies have been used to determine appropriate staffing levels for medical treatment operations, to improve the design of antibiotic distribution systems and the efficiency of vaccination programs, and to reduce operating costs. The following are samples of simulation model applications in bioterrorism preparedness and responses. Hupert (2002) used Arena simulation software package to model the overall antibiotic distribution center and determine optimal staffing levels for low, medium, and high disease-prevalence bioterrorism response. Chen (2004) aligned two fundamentally different models of smallpox transmission after a bioterrorist attack including a location-explicit multi-agent model (Biowar) and the conventional epidemiological box model (SIR). The goal of their study was to develop a greater validation process for Biowar. Another simulation model was revealed by Cantor (2004) from the Institute for Stuttering Treatment and Research (ISTAR) project, a computer simulation was used to simulate a hypothetical smallpox exposure of New Yorkers.

The preceding examples illustrate that simulation has been successfully used to develop aspects of the public health infrastructure in order to prepare for bioterrorist threats. In the current research, we concentrated on the public health systems contained within Lubbock County, Texas and its surrounding rural communities, namely Trauma Service Area-B (TSA-B). Table 1 in Appendices provides a list of healthcare units in TSA-B considered in this study. A total of ten hospitals or clinics within TSA-B healthcare systems were surveyed and the simulation models were developed individually using professional simulation software, Flexsim, version 2.6. The actual patient flow in an Emergency Room (ER) and main hospital sections were developed and incorporated to the simulation model. This simulation model can help healthcare management determine a staff and resource level in multiple hypothetical bioterrorist-attack scenarios to process high patient volumes without causing long queues and delays in the treatment process. 


\section{DESCRIPTION OF HEALTHCARE SYSTEM}

Among those hospitals in TSA-B, Texas Tech University Health Sciences Center/University Medical Center (TTUHSC/UMC) is the most comprehensive healthcare system that broadly covers treatment units, treatment functions, staff resources, and patient types. Since the simulation modeling was developed identically across all TSA-B healthcare systems, an explanation of our modeling approach is based on only the treatment process at UMC.

The UMC facility consists of an emergency room (ER) and the main hospital section. ER is staffed around the clock by doctors, triage nurses, treatment nurses, and lab physicians; while the main hospital section includes the eight treatment units of cardiac care unit (CCU), medical intensive care unit (MICU), pediatric intensive care unit (PICU), surgical intensive care unit (SICU), trauma, burn clinic, ortho and OB-GYN, with the required staffing resources.

Several different types of patients visit the hospital with a wide range of illness including urgent care patients with the majority being need a cute care, the return patient with doctor's appointment, and new patients. Most of the urgent care patients and acute care patients are sent to the hospital ER where a triage nurse determines the severity of the patients' ailment into three levels: serious, moderate, and less-severe conditions. The patients with each of

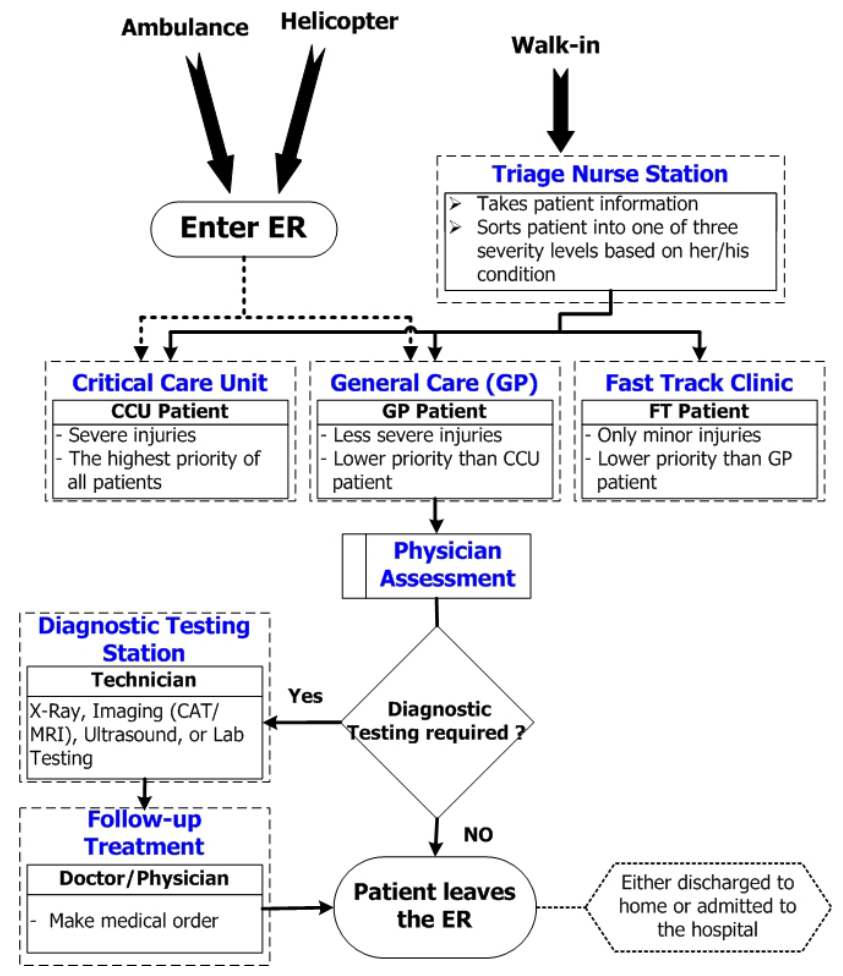

Figure 1: Patient Flowchart at Emergency Room in UMC these three levels of illness are being treated in three different treatment areas to ensure that patients with more life-threatening or painful conditions receive immediate attention. As shown in Figure 1, a flow chart of a typical ER patient's process would be similar to the following: A patient arrives in the ER waiting area for registration and triage process. When a treatment room becomes available, the patient begins an initial assessment by a nurse. Following that, the patient waits for a doctor's physical examination and an order for any further necessary testing. Then, the patient is either sent to a diagnostic lab or for consultation by other staff members if required. Upon receiving the testing results, the doctor will give further medical orders to the patient, admit the patient to the specific treatment unit within the main hospital section, or discharge the patient home.

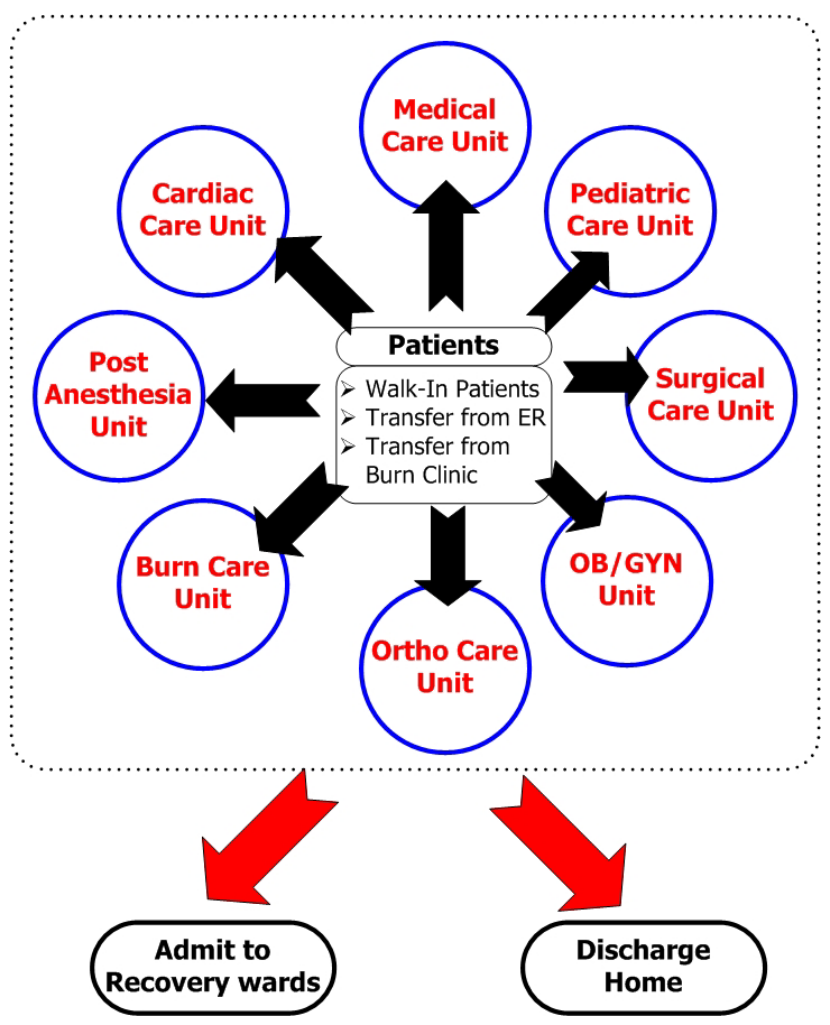

Figure 2: Patient Flowchart at Main Hospital in UMC

With the main hospital section, a patient typically enters this area through one of three modes: walk-in, admitted from the ER, or admitted from the burn clinic. In addition, the patient can be categorized as one of eight patient types depending on which treatment unit the patient receives the treatment. Although patient flow varies based on a series of activities and interactions within hospital, a typical patient's process can be described as following: A patient arrives in the admitting area for registration and determination of patient type. Following that, the patient is 
sent to the specific treatment unit (such as CCU, PICU, etc.) in order to receive the doctor's consultation. Eventually, the patient is either discharged from the hospital or transported to a corresponding patient recovery ward where accommodates the patient as directed by the doctor's orders. The big frame of a typical patient in the main hospital section is presented in Figure 2.

\section{MODELING ASSUMPTIONS}

To build a robust and representative model, certain assumptions were made to eliminate any possible misunderstandings or unrealized expectations. These model assumptions are outlined as follows:

1. Proportion of Walk-in and EMS patients (those who enter hospital by helicopter or ambulance) in each of three levels of the patients' illness (i.e. serious, moderate, and less-severe conditions).

2. Proportions of patients required to have diagnostic testing.

3. Proportion of patients who are directly discharged to home from the ER or later admitted to the hospital for further medical treatment.

4. Proportions of patients sent from the current treatment unit to the following unit, based on the patient flow.

5. Infinite capacity for doctors providing treatment in each treatment unit. However, the maximum resource capacity in each treatment unit is bounded by the number of available beds and nurses.

6. Infinite capacity for testing equipment within the diagnostic lab

\section{MODEL INPUT}

This section includes a summary of the data inputs for the simulation model. The arrival rates and the number of hospital resources were obtained from data collected by hospital record system; while the service times for all procedures and the proportion of patient types were estimated by a panel of experts familiar with the system.

\subsection{Arrival Rates}

Three main sources of patient arrivals considered in the model include ER patient, main hospital patient, and bioterrorism event arrivals. According to the current UMC's record, the ER patient arrival rate is categorized as a day time from 8:00am to 9:00pm and a night time from 9:00pm to 8:00am. Inter-arrival rates of the day time and night time were modeled as Poison process with mean of 15 and 40 minutes respectively. For the main hospital section, an inter-arrival rate of general patients was modeled as Poison process with mean of 40 minutes. To make more flexibility and allow the users to formulate the different scenarios of attack, the bioterrorism attack arrival was created to simulate the different numbers of attack days, as well as the numbers of patient arrivals on an hourly basis.

\subsection{Service Times of Treatment Process}

Since the exact service time of treatment processes can not be easily determined, a triangular distribution was used in modeling in order to allow the range of the service time as a form of the minimum, maximum, and most likely duration for treatment activities.

\subsection{Hospital Resources}

In the current study the hospital resources include both human and physical ones that provide treatment services to patients. Within ER section, the human resources consist of doctors, triage nurses, treatment nurses, and technicians; while the physical resources are the treatment rooms and beds. In the main hospital, the resources considered in this section are the number of beds, nurses, and rooms in each treatment unit. In addition, the maximum capacity in each treatment unit is also restricted by the staffing ratio between number of nurses required and number of beds provided in each treatment unit.

\subsection{Proportion of Patient Types}

This data input describes the percentage of patients in each of eight patient types, including cardiac, medical, pediatric, surgical, trauma, burn, ortho, and OB. The empirical distribution of these proportions was used to split all patient arrivals into each of eight patient types before routing them into the associated treatment units and their patient routes.

\section{SIMULATION MODEL DEVELOPMENT}

The healthcare system was modeled using Flexsim, version 2.6 to determine the total time that patients spend in the system and the hospital resource utilizations in each treatment unit of the hospital. The model was set up to gather all data inputs and recreated the overall operations of the healthcare systems in both the ER and main hospital sections using the form of an interactive model. This interactive model gives the hospital more accuracy and flexibility by allowing the analyzer to make changes in all parameters (such as the number of doctors, nurses and beds, the staffing ratios, the service time of each treatment unit, the proportion of patient type, etc.) that gets much closer to the actual system. Since Flexsim is capable of linking with Ms-Excel (Microsoft Corporation, Redmond, WA), input and output data spreadsheets have been created to give the user more visualization and ease in adjusting input data 
and analyzing output results without even knowing how Flexsim works. Moreover, the input data spreadsheet allows the user to formulate various scenarios for a bioterrorism event, hypothesizing on how many patients would be sent to the hospital on an hourly basis over the course of a number of days. The output provided by this model can contribute significantly to the level of preparedness for healthcare system, and assist in evaluating and treating the potential victims of a terrorism-related biological or chemical agent release. To give more insight to the use of the model, the user's guide was developed to demonstrate how to install Flexsim Run Time version software, define the input parameters, run the model, and analyze the model outputs.

The simulation model has been developed following the logic of typical patient flow process as described earlier and consists of two sub-models: ER and main hospital sections. Within the ER sub-model, the simulation flow was divided into three functional parts representing three different treatment areas of three levels of patients' severities, which are serious, moderate, and less-severe conditions. The patient arrivals were modeled as day time and night time inter-arrival Poison process with those values mentioned in the previous section. To model the main hospital section, we first determined the patient treatment routes for each of those eight patient types.

According to the patient flow charts provided by the hospital, a principle of the decision tree was used to determine all possible treatment routes that the patient must follow from the first treatment unit until being discharged

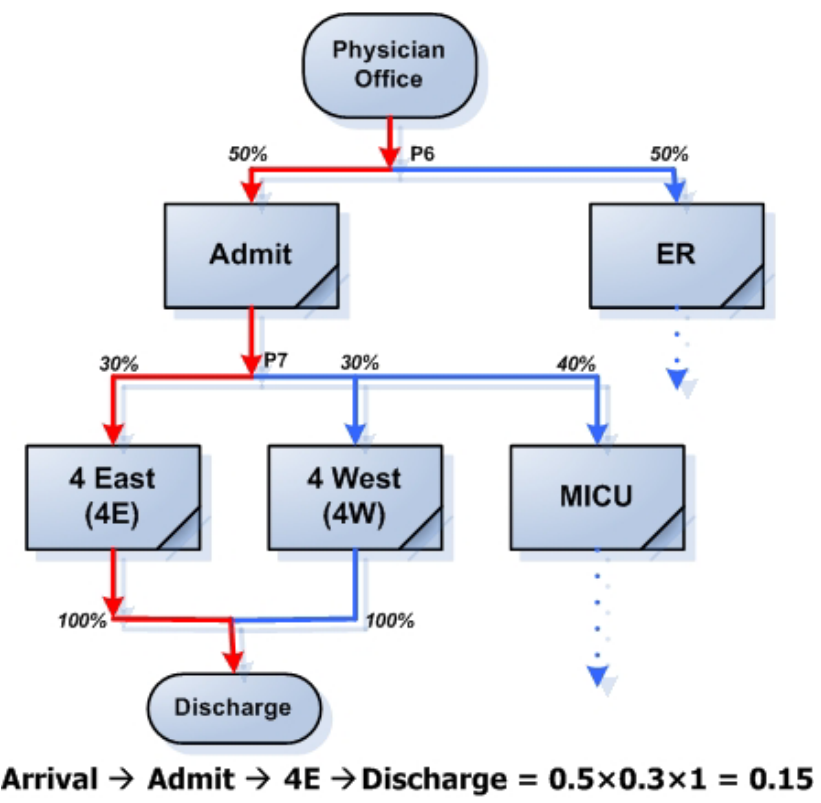

Figure 3: Example of Converting Patient Flow to Patient Route by using Decision Tree Method from the hospital. Then, the probability of having a specific route was calculated by multiplying all the corresponding percentages that patient was sent from the first treatment to the following one until discharged home. An example of determining the treatment routes and their associated probabilities is illustrated in Figure 3. Eventually, the empirical distribution was computed from these probabilities in order to assign the patients to each of possible treatment routes.

The model was validated and verified to ensure the simulation model is an accurate representation of the actual system and operates correctly as intended. The model was then run for a period of 30 working days with seven days warm-up period and results were collected. This initial model represents the overall treatment process at UMC under the current condition.

\section{SCENARIO ANALYSIS AND COMPARISON}

As mentioned at the beginning of this paper, the purpose of this study was to investigate the total time patient stays in the system, as well as the hospital resource utilizations in multiple scenarios of bioterrorism events. Once the model was validated and verified, we were able to begin an analysis of different system configurations. This paper focuses on three major scenarios: (1) current operation system, (2) medium level of a bioterrorism event, and (3) high level of a bioterrorism event.

The first scenario measured the performance of the current operation of the overall healthcare systems in both the ER and main hospital sections. The second scenario investigated how the performance of the healthcare operation changed if a medium level of a bioterrorism event happened. At this level, we assumed that there were about 100 patients a day for seven consecutive days, with an average of 4 patients an hour sent to the hospital. The last scenario is focused on finding the surge capacity, that is, the maximum patient load that the healthcare system can tolerate. At this high level, we found that the surge capacity is 960 patients a day, identical to an average of 40 patients an hour, for seven consecutive days. For this study, the response performances of interests was the overall time that it took for patients to initial enter the hospital, either in the ER or in the main hospital section, until finally being discharged. In addition, the study further looked at the utilization of resources within the hospital.

Ten replications for each of the three scenarios were run, with each replication consisting of 30 days plus a seven-day warm-up period. Figure 4 shows the total times incurred by each of eight patient types across all three scenarios. The results reveal that burn patient's staying time in the hospital is longest; following is the medical care patient and pediatric patient. In addition, Figure 5 compares the utilization of hospital resources across the three scenarios. The utilization of resources in the burn clinic, MICU, and 
PICU are the three highest utilization rates. These utilization results are clearly proportional to the results of the total time patient spent. Therefore, it is a confident conclusion that the burn treatment area, MICU, and PICU represent the process bottlenecks in the hospital.

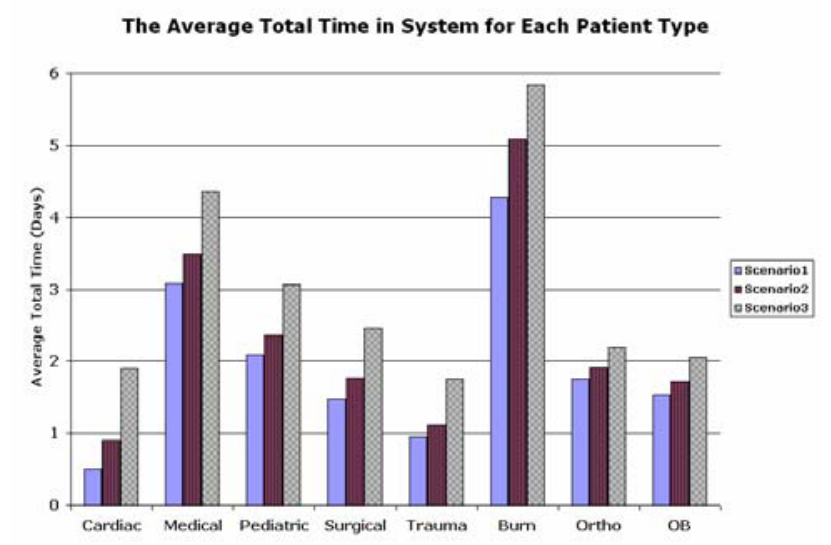

Figure 4: Patients Total Staying Time in System across Three Scenarios

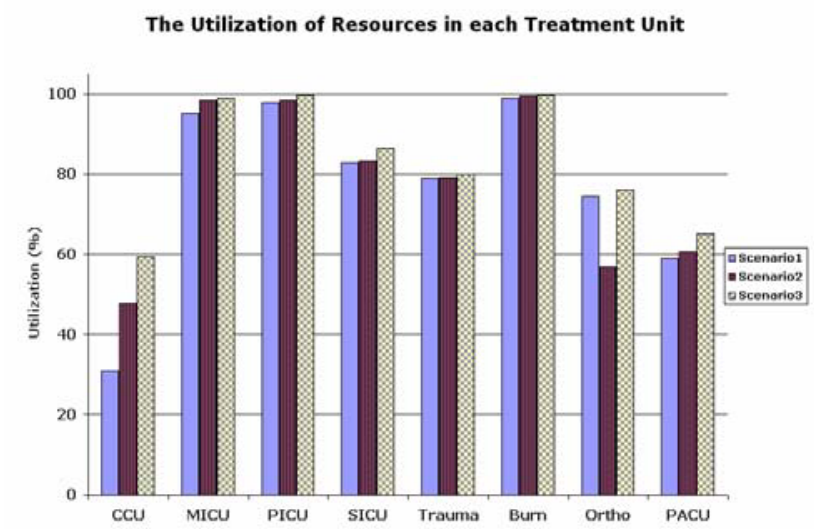

Figure 5: Hospital Resources Utilization across Three Scenarios

By comparing the outputs of the three scenarios, we were able to draw the conclusions. These results are evident that the different scenarios provide different mean total patient times in the system. As initial suspected, the total time significantly increases when there are more patients entering the hospital in the event of bioterrorism. The lower the level of bioterrorism, the smaller the total time a patient spends in the system. In the current research hospital resource utilization was defined as a percentage of the working time divided by the total simulation time that hospital resources are occupied toward the medical treatment, excluding all breaks and allowances, which can account for only a very small proportion. As expected, the simulation result shows that the utilization of resources in the current system is smaller than those in a bioterrorism event. The addition of patient arrival in a bioterrorism event causes the utilization levels of most hospital resources higher; although all service times remain the same. When the number of patient arrivals is increased to the surge capacity, the utilization rates of hospital resources in PICU and burn clinic areas reach a level of $100 \%$, causing a crash of the model.

\section{CONCLUSION AND FUTURE WORK}

The simulation models using Flexsim software for the overall treatment process at Lubbock area healthcare systems in Texas were individually developed, validated, verified, and implemented to aid in decision-making about surge capacity preparedness in the event of bioterrorism. With the big frame of the UMC model representing the prototype hospital in this paper, the model can be visibly divided into two components: the Emergency Room (ER) and the main hospital. While patients can arrive at the ER in one of only two ways-- private car (walk-in) or Emergency Medical Service (EMS) -- patient arrivals to the main hospital come from the following three sources: walk-in, admitted patient from the ER or admitted patient from the burn clinic. Thus, some ER patients who are admitted to the hospital represent the output of the initial ER component and are later converted into an input for the main hospital component. The characteristic that distinguishes our model from the existing healthcare simulation models is that it is highly flexible. The model is easily customized, allowing the user to make changes to all input parameters via the user interface of an Excel file without having to access the simulation software or language at any time. The user's guide also allows the users to easily utilize the model.

The simulation model was run with three scenarios, including the current operation system, medium level of a bioterrorism event, and high level of a bioterrorism event at the level of a surge capacity. The performance measures of interests were the total time that patient stays in the system and the utilization of hospital resources. The output results from these three scenarios suggest that the higher number of patient arrivals lead to the higher total time that patients spend in the system and therefore the higher rate of resource utilization. In addition, when the number of patients reaches the surge capacity of 960 patients a day (or an average of 40 patients an hour) for seven consecutive days addition to the current patient arrival level of the ER and the main hospital, some hospital resources reach the level of $100 \%$ utilization, causing the simulation model to crash.

The benefit of our simulation model is to allow the hospital managements to formulate multiple possible scenarios for bioterrorism events and evaluate the preparedness of their hospital resources. The possible future work 
includes the use of data obtained from the simulation model to perform the staffing coverage or an analysis of location-routing problems of medical distribution among all hospitals in surrounding area for the event of bioterrorism attacks.

\section{ACKNOWLEDGMENTS}

The support of this research by Trauma Service Area-B Regional Advisory Council (BRAC) in Lubbock, Texas, is gratefully acknowledged.

\section{A APPENDICES}

Table 1: A list of the Healthcare Systems in TSA-B in the Current Research.

\begin{tabular}{|c|l|c|}
\hline No. & \multicolumn{1}{|c|}{ Healthcare Unit } & Location (County) \\
\hline 1 & $\begin{array}{l}\text { Brownfield Regional } \\
\text { medical Center }\end{array}$ & Brownfield \\
\hline 2 & $\begin{array}{l}\text { Cochran Memorial } \\
\text { Hospital }\end{array}$ & Morton \\
\hline 3 & $\begin{array}{l}\text { Covenant Medical Center } \\
\text { at Lubbock }\end{array}$ & Levelland \\
\hline 4 & $\begin{array}{l}\text { Covenant Medical Center } \\
\text { at Levelland }\end{array}$ & Crosbyton \\
\hline 5 & $\begin{array}{l}\text { Crosbyton Clinic } \\
\text { Hospital }\end{array}$ & Littlefield \\
\hline 6 & Lamb Healthcare & Lubbock \\
\hline 7 & Lubbock Heart Hospital & Lamesa \\
\hline 8 & Medical Arts Hospital & Lubbock \\
\hline 9 & $\begin{array}{l}\text { University Medical Cen- } \\
\text { ter at Texas Tech } \\
\text { University }\end{array}$ & Lockney \\
\hline 10 & W.J. Mangold Memorial & \\
\hline
\end{tabular}

\section{REFERENCES}

Cantor, S. (2004). Project Models Virus Outbreak, Spread. The Daily Pennsylvanian, University of Pennsylvania, Pennsylvania.

Chen, L., Kaminsky, B., Tummino, T., et al. (2004). Aligning Simulation Models of Smallpox Outbreaks. The 2nd NSF/NIJ Symposium on Intelligence and Security Informatics (presentation).

Hupert, N., Mushlin, A.I., and Callahan, M.A. (2002). Modeling the Public Health Response to Bioterrorism: Using Discrete Event Simulation to Design Antibiotic Distribution Centers. Medical Decision Making, Vol. 22 supplementary, pp. S17-S25.

Welter, D.C., Daniell, W.E., and Treser, C.D. (2001). Hospital Preparedness for Victims of Chemical or Biological Terrorism. American Journal of Public Health, Vol. 91, No. 5, pp. 710-716.

\section{AUTHOR BIOGRAPHIES}

LISA PATVIVATSIRI has been working as an applications engineer in area of production scheduling and simulation modeling at PMC. She receives a doctoral degree in the Department of Industrial Engineering at Texas Tech University. She has a M.S. in Industrial and Systems Engineering from Virginia Polytechnic Institute and State University (Virginia Tech) and a BS degree in Industrial Engineering from Chulalongkorn University in Thailand. Her research interests include simulation modeling and scheduling in the healthcare and manufacturing industries.

ELLIOT J. MONTES, JR. has been working in the simulation modeling environment at W.L. Gore and Associate. He has previously held positions in the Department of Industrial Engineering at the Texas Tech University. His research interest lies in the area of discrete-event simulation, scheduling problem, and simulation optimization.

OUYANG XI has been working in design plan reviewing for American Bureau of Shipping. He received his Ph.D. degree in Industrial engineering at Texas Tech University in Lubbock, Texas. He has a M.E. degree in Industrial Engineering at Lamar University, Beaumont, Texas, and a B.S. degree in Mechanical Engineering at Tianjin University, Tianjin, China. His research interests are focused on multiple objective optimization and large scale simulation. $\mathrm{He}$ is also interested in environmentally conscious product design and manufacturing. 